\title{
Ferramenta ontoKEM: uma contribuição à Ciência da Informação para o desenvolvimento de ontologias
}

\section{Sandro Rautenberg}

\author{
Professor do Depto de Ciência da \\ Computação/UNICENTRO.Doutor em Engenharia \\ e Gestão do Conhecimento EGC/UFSC
}

Antonio Costa Gomes Filho

Professor do Depto de Administração/UNICENTRO.

Doutorando em Engenharia e Gestão do

Conhecimento - EGC/ UFSC

José Leomar Todesco

\section{Professor do Programa de Pós-Graduação em Engenharia e Gestão do Conhecimento - EGC/UFSC}

Fernando Álvaro Ostuni-Gauthier

\begin{abstract}
Professor do Programa de Pós-Graduação em Engenharia e Gestão do Conhecimento EGC/UFSC
\end{abstract}

No campo da Ciência da Informação, ontologias tem sido desenvolvidas e aplicadas para organizar o conhecimento. Neste contexto, munir-se de metodologias e ferramentas adequadas em trabalhos de desenvolvimento de ontologias é pertinente. Este artigo trata da ferramenta para o desenvolvimento de ontologias ontoKEM, na perspectiva de sua contribuição à Ciência da Informação. Conclui-se que, mediante um processo metodológico incorporado, os artefatos de documentação e exemplos de aplicação da ontoKEM tem sua utilidade perante outros estudos na Ciência da Informação.

Palavras-chave: ontoKEM; Ontologias; Ciência da Informação; Ferramentas para o desenvolvimento de ontologias; Ferramentas para a Ciência da Informação. 


\title{
The ontoKEM tool: a contribution for Information Science to the ontology's development
}

\begin{abstract}
In the Information Science field, ontologies are developed and applied to organize knowledge. In this context, taking advantages from using appropriate tools and methodologies to develop ontologies becomes relevant. This article discusses about an ontology development tool, the ontoKEM, taking into account its contribution to the Information Science domain. Through an incorporated methodology and its rich range of document artifacts, we concluded that ontoKEM is useful to many Information Science studies.
\end{abstract}

Keywords: ontoKEM; Ontologies; Information Science; Tool for ontology development; Tools for Information Science.

Recebido em 22.04.2009 Aceito em 05.03.2010

\section{Introdução}

Os anos noventa do século $X X$ foram marcados por uma nova era nas Tecnologias de Informação e Comunicação, em que a Internet teve sua forma de utilização ampliada para outros fins (LIAO, 2003), além da pesquisa científica. Isso contribuiu para a geração de novos instrumentos capazes de, efetivamente comunicar e compartilhar informações (ISKE; BOERSMA, 2005). Nesse contexto, insere-se a Ciência da Informação (CI) como a principal área de conhecimento responsável pela padronização da terminologia utilizada para se encontrar e se classificar informações (ALMEIDA, 2003), auxiliando nas atividades de comunicação e compartilhamento do conteúdo informacional.

Nesse cenário, considerando-se que o número crescente e volumétrico das fontes de dados disponíveis dificulta a recuperação da informação, os estudos sobre a organização da informação têm se tornados pertinentes (ALMEIDA; BAX, 2003), sendo que em tais estudos 0 uso de instrumentos tecnológicos geralmente é considerado (CAMPOS et al., 2003; ALMEIDA; BAX, 2003) e necessário. Neste sentido, Moreira (2002) e Almeida (2003) afirmam que um instrumento de utilização em particular tem atraído o interesse dos pesquisadores da CI para as atividades de modelagem e recuperação de informação (GOTTSCHALGDUQUE, 2005). Este instrumento é denominado ontologias. Conforme 
Pickler (2007), por meio do uso de ontologias será possível elaborar uma grande rede de conhecimento, contemplando o processamento de máquina e melhorando qualitativamente os serviços web, sobretudo, os serviços de busca e recuperação de dados.

Por outro lado, em se tratando do desenvolvimento de ontologias, vários métodos e ferramentas têm sido propostos (CORCHO et al., 2003), o que para Almeida e Bax (2003) tem dificultado a escolha e a utilização do ferramental adequado à manipulação de ontologias na organização da informação. Entretanto, como a criação de ontologias geralmente envolve processos manuais (GOTTSCHALG-DUQUE, 2005), apoiar-se no seu desenvolvimento pode representar ganhos significativos (ALMEIDA; BAX, 2003).

Atendo-se aos processos manuais do desenvolvimento de ontologias, percebe-se que o processo de documentação é tido como crucial (PINTO; MARTINS, 2004). Porém, Almeida (2006) afirma que não há um consenso no processo de documentação de ontologias e dentre as metodologias $^{1}$ para ontologias não existe uma para documentar o desenvolvimento. Por outro lado, o autor recomenda que a obtenção de documentos de cada fase do ciclo de vida da ontologia é pertinente. Isso corrobora a visão de Pinto e Martins (2004), os quais afirmam que a documentação de uma ontologia é relevante, não somente para melhorar a clareza desta, mas também para facilitar nas atividades de manutenção, uso e reuso.

Por isso, considerando-se a diversidade de metodologias e a dificuldade da escolha do ferramental adequado para o desenvolvimento de ontologias, pressupõe-se que o uso de ferramentas que unificam as principais vantagens de algumas metodologias de desenvolvimento de ontologias, gera automaticamente os artefatos de documentação, sendo útil no âmbito da CI. Dito isso, este artigo discute a ferramenta ontoKEM (ONTOlogies for Knowledge Engineering and Management), como uma contribuição à CI, para desenvolver ontologias na perspectiva do auxilio à organização e recuperação da informação. Destaca-se, de antemão, que as características principais da ontoKEM são: i) incutir um método de desenvolvimento de ontologias baseado em vantagens metodológicas da guia Ontology Development 101 (NOY; MCGUINNESS, 2008) e das metodologias On-to-Knowledge (SURE; STUDER, 2003) e METHONTOLOGY (GÓMEZ-PÉREZ et al., 2004); e ii) gerar, automaticamente, uma gama de artefatos de documentação (RAUTENBERG et al., 2008). Tem-se em mente que tais características são requisitos importantes de projetos de ontologias no campo da CI.

Para tanto, além desta seção introdutória, este artigo apresenta: i) a fundamentação teórica, definindo a CI e as ontologias e discorrendo sobre a pertinência do desenvolvimento de ontologias no âmbito da $\mathrm{CI}$; ontoKEM, evidenciando suas concepção, metodologia incorporada, gama

\footnotetext{
${ }^{1} \mathrm{Na}$ perspectiva de englobar e integrar uma série de técnicas ou de métodos para criar uma teoria geral e sistêmica de como realizar uma classe de trabalho intensivo de conhecimento (IEEE, 1995).
} 
de documentação e limitações; iii) os casos de aplicação da ontoKEM em pesquisas aplicadas de desenvolvimento de ontologias no campo da $\mathrm{CI}$; e as conclusões e os trabalhos futuros.

\section{Fundamentação teórica}

Para Gasevic, Djuric e Deved (2006), armazenar conhecimento é um processo de registrar conhecimento na memória do computador, em um formato adequado. Recuperar conhecimento é um processo inverso encontrar o conhecimento quando este é necessário. Contudo, um importante pré-requisito a este processo é a aquisição do conhecimento que visa coletar, organizar e estruturar o conhecimento sobre um domínio. Tais assertivas remetem ao território familiar de práticas da área denominada CI.

Na percepção de Gomes Filho, Damásio e Miranda (2000) o objeto de estudo da CI é campo controverso quando se tenta estabelecer seu domínio, mas citam, dentre outros, que seus estudos podem ser em tecnologias da informação, planejamento de sistemas de informação, organização de bases de dados. Segundo Moreira (2002), tradicionalmente, a CI tem como objeto de estudo a classificação do conhecimento, visando o desenvolvimento de métodos classificatórios que facilitem o armazenamento e a busca de informações. Assim o sendo, Marcondes et al (2008) mostram que a CI tem especial interesse no registro do conhecimento e nas diferentes formas de representá-lo, para permitir a sua apropriação social, ou seja, sua recuperação pelo usuário final. Para os referidos autores, este interesse tem evoluído em direção à representação de conhecimento em formatos legíveis por computador.

Inserindo ontologias nesse contexto, é observado que um grande volume de pesquisa no campo da CI tem sugerido a importância e a utilização destas na tarefa de organizar informações (MOREIRA, 2002; ALMEIDA; BAX, 2003). Isso é justificado pelo fato de que as ontologias: i) propiciam melhorias na recuperação da informação, ao organizar o conteúdo de fontes de dados que compõem um domínio (ALMEIDA, 2003; MARCONDES et al., 2008) e ii) utilizam formas de representação baseadas em lógica, o que possibilita o uso de mecanismos de inferência para criar novo conhecimento a partir do existente (MARCONDES et al., 2008).

É importante ressaltar que os estudos originais sobre ontologias são do campo da Filosofia e, recentemente, estendidos aos de representação de conhecimento (KIRYAKOV, 2006). No sentido do artefato de representação do conhecimento, para Uschold (1998) uma ontologia pode assumir várias formas. Contudo, ela necessariamente deve incluir um vocabulário de termos e alguma especificação do significado desses termos. Isto inclui definições e alguma indicação de como os conceitos estão inter-relacionados, o que repercute tanto na imposição da estrutura do domínio e em restrições, quanto na interpretação dos termos. 
Formalmente, uma ontologia representa um domínio através do relacionamento de quatro elementos, representado por $O=\{C, R, I, A\}$ donde (KIRYAKOV, 2006):

i. C: é o conjunto de classes que representam os conceitos em um dado domínio de interesse;

ii. R: é o conjunto de relações ou associações entre os conceitos do domínio;

iii. I: é o conjunto de instâncias derivadas das classes, ou ainda, os exemplos concretos de classes conceituais representados em uma ontologia;

iv. A: é o conjunto de axiomas do domínio, que servem para modelar restrições e regras inerentes às instâncias.

Tecnicamente, o relacionamento entre esses quatro elementos permite que as definições dos conceitos de um domínio e o relacionamento entre essas definições sejam tratadas computacionalmente (DACONTA; OBRST; SMITH, 2003). Desta forma, as ontologias são utilizadas como meio para comunicação de conhecimento, integração de conhecimento e inferência com conhecimento (MIKA; AKKERMANS, 2005). Na CI, tais empregos apontam, por exemplo, para a expansão do poder de recuperação de informação dos tesauros (ALMEIDA; BAX, 2003).

Contudo, para se desenvolver ontologias é necessário um esforço considerável de engenharia, disciplina e rigor, no qual princípios de projeto, atividades e processos de desenvolvimento, tecnologias de suporte e metodologias sistêmicas devem ser empregados (GASEVIC; DJURIC; DEVED, 2006).

Corroborando, Gómez-Pérez et al. (2004) salientam que o desenvolvimento de ontologias é uma atividade complexa e que consome muito tempo e recursos (GÓMEZ-PÉREZ et al., 2004), sendo que essa atividade necessita ser apoiada de alguma forma (ALMEIDA; BAX, 2003). Neste sentido, há uma riqueza de ferramentas (GÓMEZ-PÉREZ et al., 2004) e de metodologias (CORCHO et al., 2003) voltadas ao desenvolvimento de ontologias, cada qual com suas características ao se considerar todo o ciclo de especificação, conceitualização, formalização, implementação e manutenção de uma ontologia.

Para Almeida e Bax (2003), essa riqueza, anteriormente relatada, tem dificultado a escolha e a utilização do ferramental adequado à manipulação de ontologias na organização da informação. Uma forma de tentar dirimir isso é se instrumentalizar de uma combinação de metodologias, pautando-se nas vantagens e nos artefatos de cada uma das metodologias (FERNANDEZ-LÓPEZ; GÓMEZ-PÉREZ, 2002; SURE; STUDER, 2003; BRUSA et al., 2008). Essa foi a inspiração principal do desenvolvimento da ferramenta ontoKEM, como discutido a seguir. 


\section{A ferramenta ontoKEM}

Ilustrada na FIG. 1, a ontoKEM ${ }^{2}$ é uma ferramenta case baseada na web de propósito acadêmico para documentação e construção de projetos de ontologias.

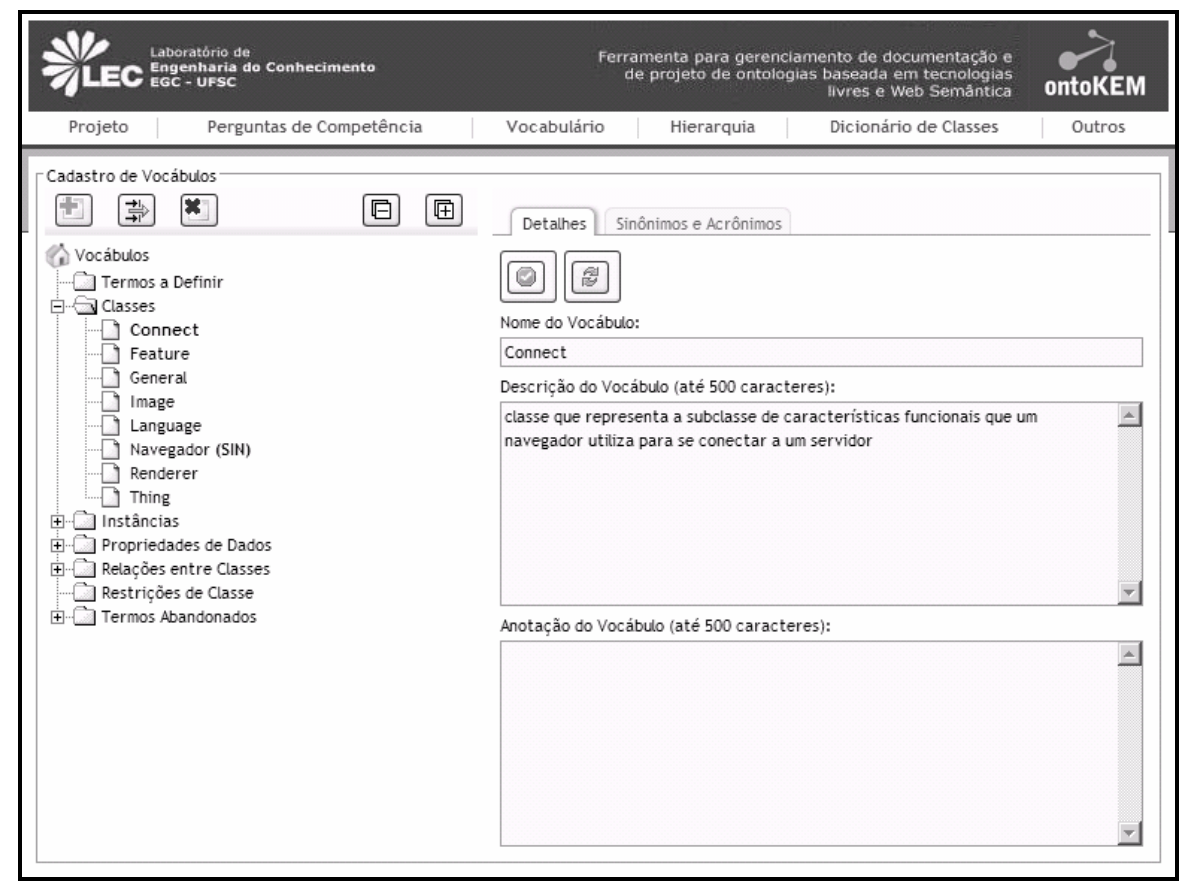

FIGURA 1 - Tela do ontoKEM - módulo cadastro de vocábulos -Fonte: (ONTOKEM, 2009).

\subsection{A concepção da ontoKEM}

A concepção da ontoKEM se deu no início de 2007 e, originalmente, pela necessidade de se garantir eficiência ao processo de ontologia em um projeto de pesquisa e desenvolvimento denominado "Gestão da Rede de Distribuição de Média Tensão Empregando Ontologias de Domínio e Sistemas de Conhecimento - Programa de Pesquisa e Desenvolvimento CELESC Ciclo 2005-2006" (RELATÓRIO..., 2008). Nesse projeto, de forma colaborativa, a ontoKEM suportou a primeira fase dos trabalhos dos especialistas de domínio do setor elétrico e dos engenheiros do conhecimento envolvidos na construção da ontologia. Mais precisamente, a ferramenta foi empregada nas atividades de especificação e aquisição do conhecimento (RAUTENBERG et al., 2008).

\footnotetext{
${ }^{2} \mathrm{O}$ desenvolvimento da ontoKEM é realizado e coordenado por membros do LEC/EGC/UFSC. Participa da equipe um pesquisador do Laboratório de Inteligência Computacional e Pesquisa Operacional (UNICENTRO). A utilização da ferramenta tem como objeto a pesquisa aplicada/exploratória. Demais informações são acessadas em http://ontokem.egc.ufsc.br.
} 
Com base nas experiências geradas e nos avanços na implementação da ferramenta, no segundo semestre do mesmo ano, a ontoKEM passou a ser empregada como suporte ao ensino de uma metodologia de desenvolvimento de ontologias na disciplina de Tópicos Especiais em Engenharia do Conhecimento do Programa de PósGraduação em Engenharia e Gestão do Conhecimento da Universidade Federal de Santa Catarina, sendo também empregada na reoferta da disciplina. Vale ressaltar que dentre os discentes da referida disciplina, alguns eram oriundos da área da CI.

Nas experiências do emprego da ferramenta, comparado a outras ferramentas, cabe ressaltar que o grande apelo da ontoKEM reside na incorporação de um método de desenvolvimento de projetos de ontologias e, principalmente, na gama de artefatos de documentação gerados de forma automática durante o desenvolvimento. Pressupõe-se que tais características são importantes na construção de ontologias também no domínio da CI e, nessa linha de raciocínio, a ontoKEM é uma contribuição à CI.

A seguir, são relatados o processo metodológico e os artefatos de documentação da ontoKEM.

\subsection{A metodologia incorporada na ontoKEM}

Em se tratando de metodologias para desenvolvimento de ontologias, Corcho et al. (2003) afirmam existirem metodologias que são empregadas em tarefas específicas no ciclo de vida de uma ontologia. Corroborando, para Fernandez-López e Gómez-Pérez (2002), em cada metodologia proposta existem atividades que deixam de estar compreendidas. Ainda, para Sure e Studer (2003) e Brusa et al.(2008), uma combinação de metodologias se torna pertinente no desenvolvimento de ontologias. Partindo-se desta premissa, a seguir são descritas, resumidamente, as metodologias utilizadas como subsídios para a composição do processo metodológico incorporado na ontoKEM.

\section{Guia Ontology Development 101}

Segundo Noy e McGuinness (2008), não existe um modo correto ou metodologia de desenvolvimento de ontologias. Por isso, os autores sugerem apenas um processo para tal, denominado Ontology Development 101. Este processo consiste em um guia de passos iterativos, livremente executados no desenvolvimento de ontologias. A FIG. 2 ilustra (a) os sete passos sugeridos pelos pesquisadores e (b) um exemplo de como os passos podem ser empregados durante 0 desenvolvimento de uma ontologia. Resumidamente, os sete passos do guia Ontology Development 101 são: determinar o escopo da ontologia, considerar o reuso de ontologias desenvolvidas, enumerar os termos do domínio, definir as classes do domínio e sua hierarquia, definir as 
propriedades das classes (de dados ou relações), definir as restrições das propriedades e criar instâncias.

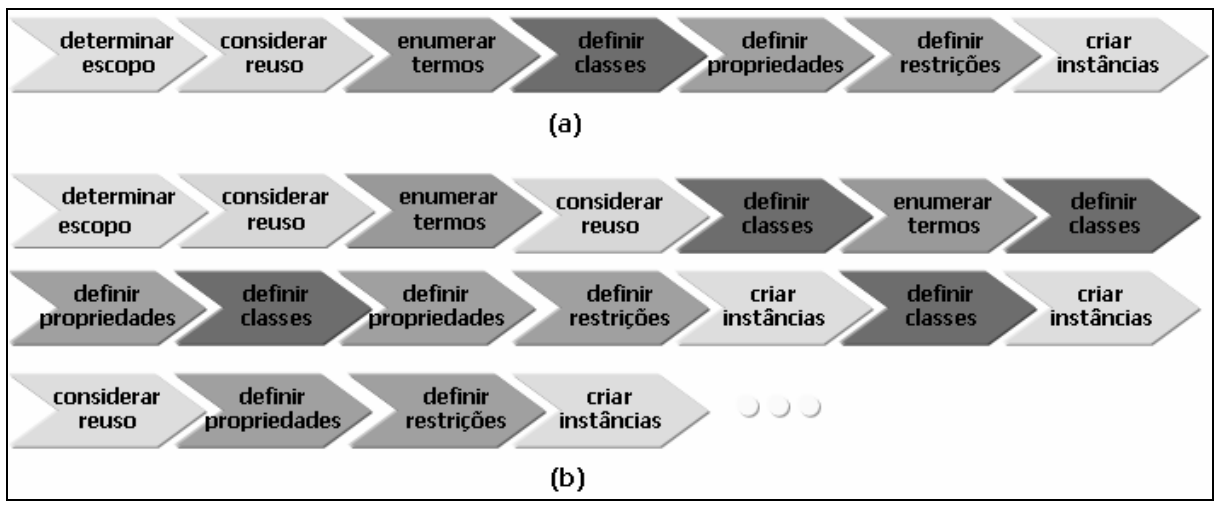

FIGURA 2 - Processo de desenvolvimento no guia Ontology

Fonte: baseado em NOY; MCGUINNESS, 2008.

Perante o processo metodológico incutido na ontoKEM, o guia Ontology Development 101 contribui com uma visão clara de como se dá um processo iterativo para o desenvolvimento de ontologias.

On-to-Knowledge

On-to-Knowledge é uma metodologia de desenvolvimento de ontologias, fruto da cooperação de várias entidades européias (FENSEL; HERMELEN, 2008), tendo como intuito desenvolver ontologias para serem empregadas em Sistemas de Gestão do Conhecimento. Conforme mostra a FIG. 3, essa metodologia é dividida em cinco fases (SURE; STUDER, 2003), sendo elas: estudo de viabilidade, início da ontologia, refinamento, avaliação, manutenção e evolução.

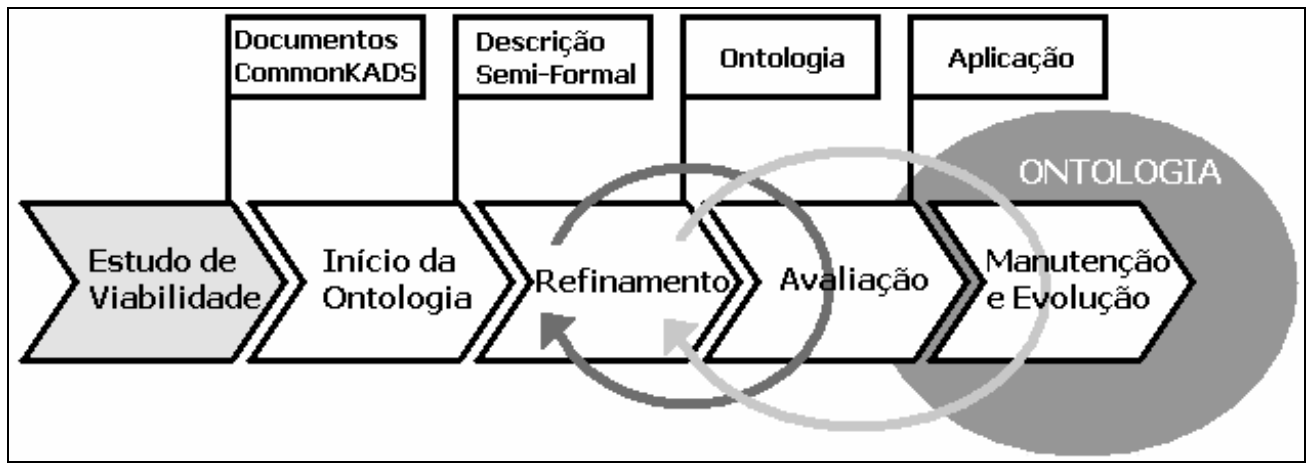

FIGURA 3 - Representação da metodologia On-to-Knowledge Fonte: adaptado de SURE; STUDER, 2003.

A característica principal do On-to-Knowledge para com a ontoKEM é sua preocupação com as fases iniciais do estudo de viabilidade e início da ontologia. Nesse sentido, é clara a necessidade de se definir o domínio e o 
escopo da ontologia, sobretudo, na utilização de questões de competência para tal delineamento. Documentos de escopo da ontologia e de questões de competência estão dentre os artefatos de documentação da ontoKEM.

Methontology

METHONTOLOGY é uma metodologia de desenvolvimento de ontologias idealizada por um grupo de pesquisa em Engenharia de Ontologias da Universidade Politécnica de Madri, sendo fortemente influenciada por metodologias de engenharia de software e de engenharia do conhecimento (GÓMEZ-PÉREZ et al., 2004). A METHONTOLOGY prevê um ciclo de vida baseado na prototipagem de ontologias, de acordo com a evolução do processo de desenvolvimento (especificação, conceitualização, formalização, implementação e manutenção). Inspirando-se em preceitos do ciclo de vida de software, as atividades privilegiadas na METHONTOLOGY são destinadas ao gerenciamento, desenvolvimento e suporte do ciclo de vida de uma ontologia, conforme FIG. 4.

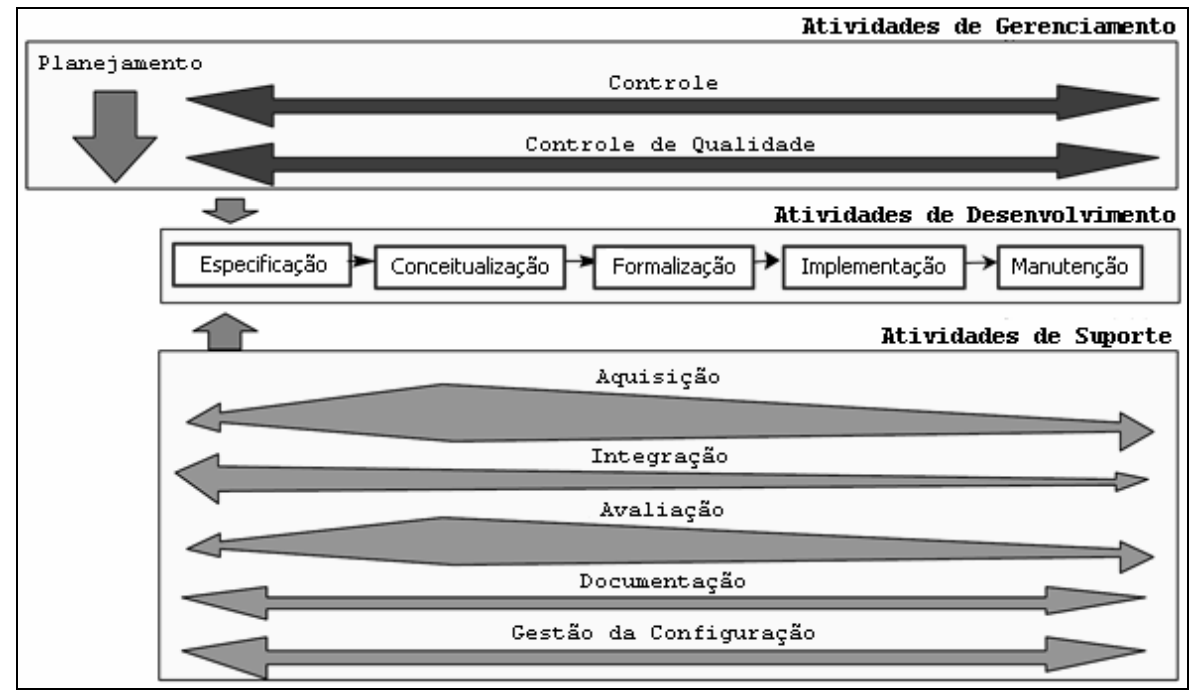

FIGURA 4 - Representação da metodologia METHONTOLOGY Fonte: adaptado de GÓMEZ-PÉREZ et al., 2004.

Considerando-se as demais metodologias de desenvolvimento de ontologias, a característica principal da METHONTOLOGY é a definição de um rico conjunto de artefatos de documentação, dos quais a ontoKEM também se utiliza. 
Ferramenta ontoKEM: uma contribuição à Ciência da Informação para o desenvolvimento de ontologia
Sandro Rautenberg; Antonio Costa Gomes Filho; José Leomar Todesco; Fernando Álvaro Ostuni-Gauthier

QUADRO 1- Contribuições das metodologias à ferramenta ontoKEM.

\begin{tabular}{|c|l|}
\hline Metodologia & \multicolumn{1}{c|}{ Contribuição } \\
\hline Ontology Development 101 & processo iterativo para o desenvolvimento de ontologias \\
\hline On-to-knowledge & $\begin{array}{l}\text { definição do domínio e o escopo da ontologia; e utilização } \\
\text { de questões de competência }\end{array}$ \\
\hline METHONTOLOGY & rico conjunto de artefatos de documentação \\
\hline
\end{tabular}

Fonte: elaborado pelos autores.

O QUADRO 1 resume as contribuições de cada uma das metodologias citadas no processo metodológico incorporado na ontoKEM. A seguir, apresenta-se os artefatos de documentação gerados pela ontoKEM.

\subsection{Os artefatos de documentação da ontoKEM}

Esta subseção discute os artefatos de documentação gerados pela ontoKEM durante o desenvolvimento de uma ontologia. Para exemplificar os documentos, utiliza-se como referência central, a ontologia de domínio desenvolvida por Molossi (2008), que se utilizou da metodologia ontoKEM (ONTOKEM, 2009) para aplicação à Ciência da Informação.

Seguindo o processo metodológico incorporado pela ontoKEM, o primeiro artefato de documentação oferecido pela ferramenta é o "Escopo da Ontologia", tal qual representado na FIG. 5. O intuito na disponibilização deste artefato é registrar respostas às questões sobre a abrangência do domínio e a utilidade da ontologia.

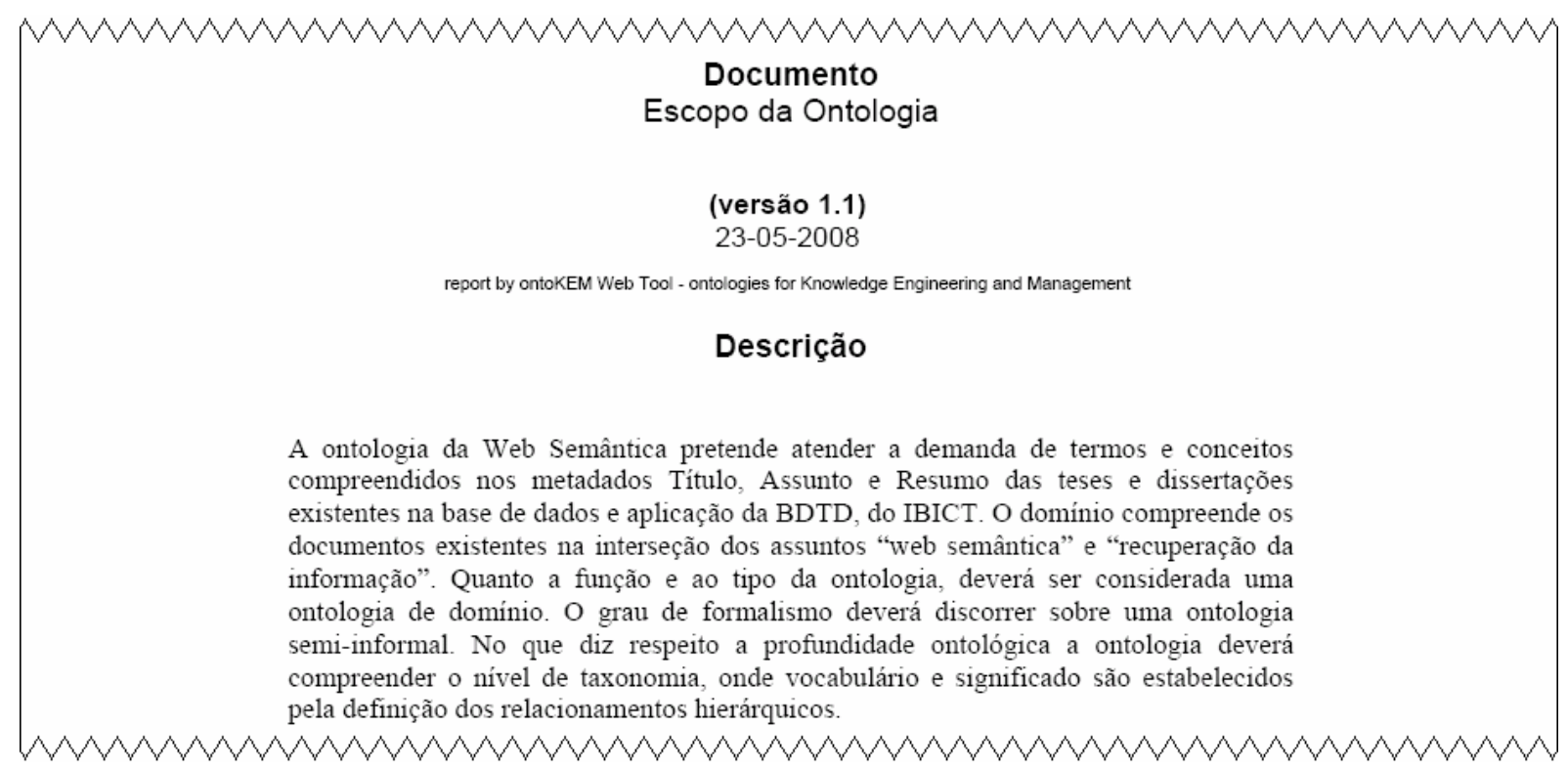

FIGURA 5 - Representação do documento "Escopo da Ontologia"

Fonte: adaptado de MOLOSSI, 2008. 
Em se definindo o escopo da ontologia, o próximo passo importante incorporado à ontoKEM é a definição das questões de competência. A definição das questões de competência é um modo simples e direto de se confirmar o escopo de uma ontologia, permitindo-se identificar, antecipadamente, conceitos, propriedades, relações e instâncias. Para amparar esse processo, a ontoKEM disponibiliza 0 artefato de documentação "Questões de Competência" (FIG. 6), o qual enumera as questões de competência da ontologia, listando-se seus respectivos termos e relações.

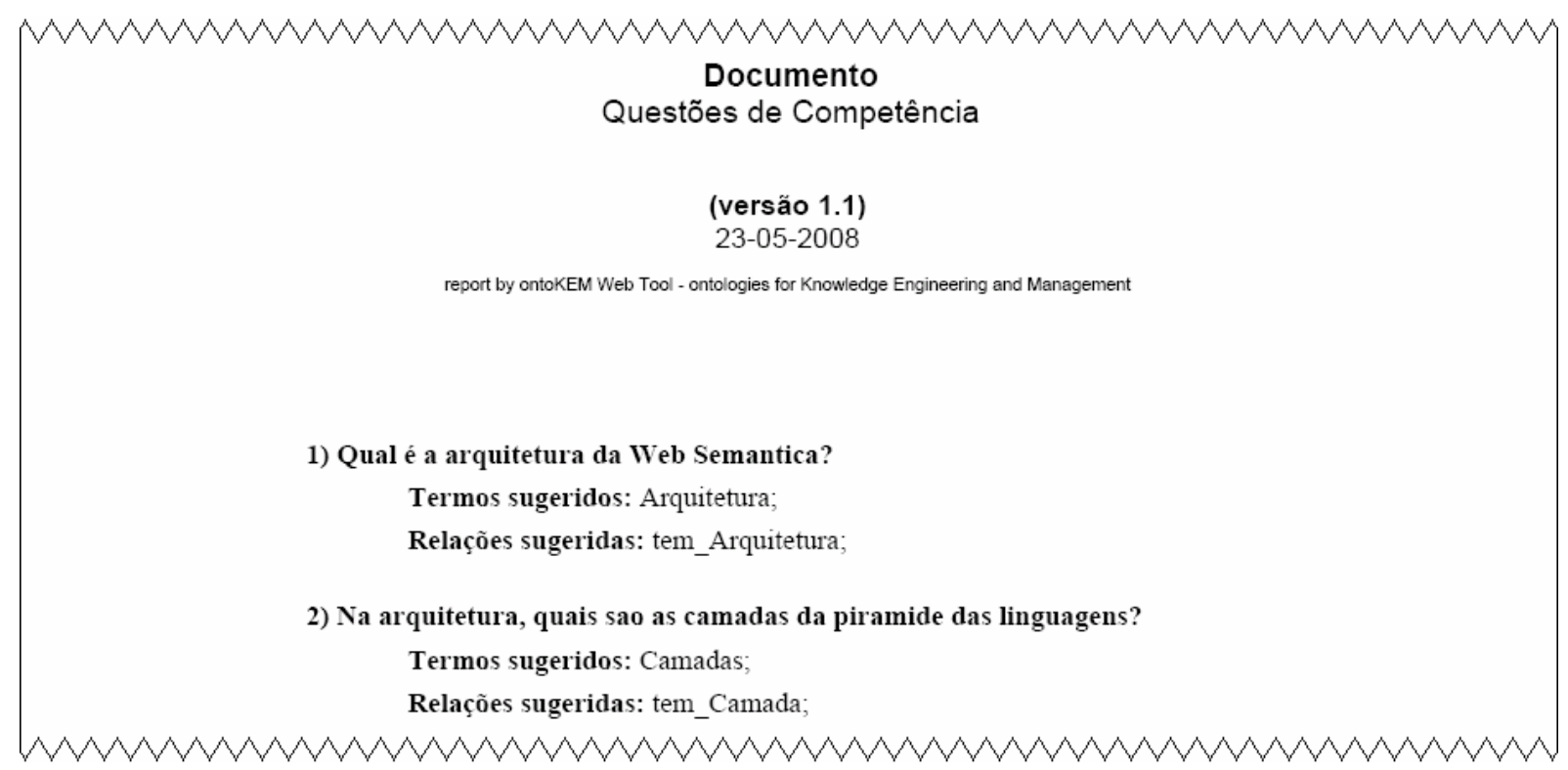

FIGURA 6 - Representação do documento "Questões de Competência" Fonte: adaptado de MOLOSSI, 2008.

Como as ontologias se constituem a partir do entendimento compartilhado sobre a representação de um domínio, uma fase importante do desenvolvimento é o consenso a respeito dos elementos do domínio. Para corroborar o consenso, a ontoKEM provê um artefato de documentação denominado "Vocabulário Completo" (FIG. 7). Nesse documento, todos os elementos do domínio são classificados como classes, relações, propriedade de dados, instâncias e restrições e tem sua definição apresentada. Tal documento pode transitar entre engenheiros de ontologias e especialistas de domínio, auxiliando no refinamento consensual das definições. Salienta-se que esse documento pode ser particularizado para enumerar somente classes, ou relações, ou propriedades de dados, ou restrições, ou instâncias mediante opções disponibilizadas na ferramenta. 
Ferramenta ontoKEM: uma contribuição à Ciência da Informação para o desenvolvimento de ontologia
Sandro Rautenberg; Antonio Costa Gomes Filho; José Leomar Todesco; Fernando Álvaro Ostuni-Gauthier

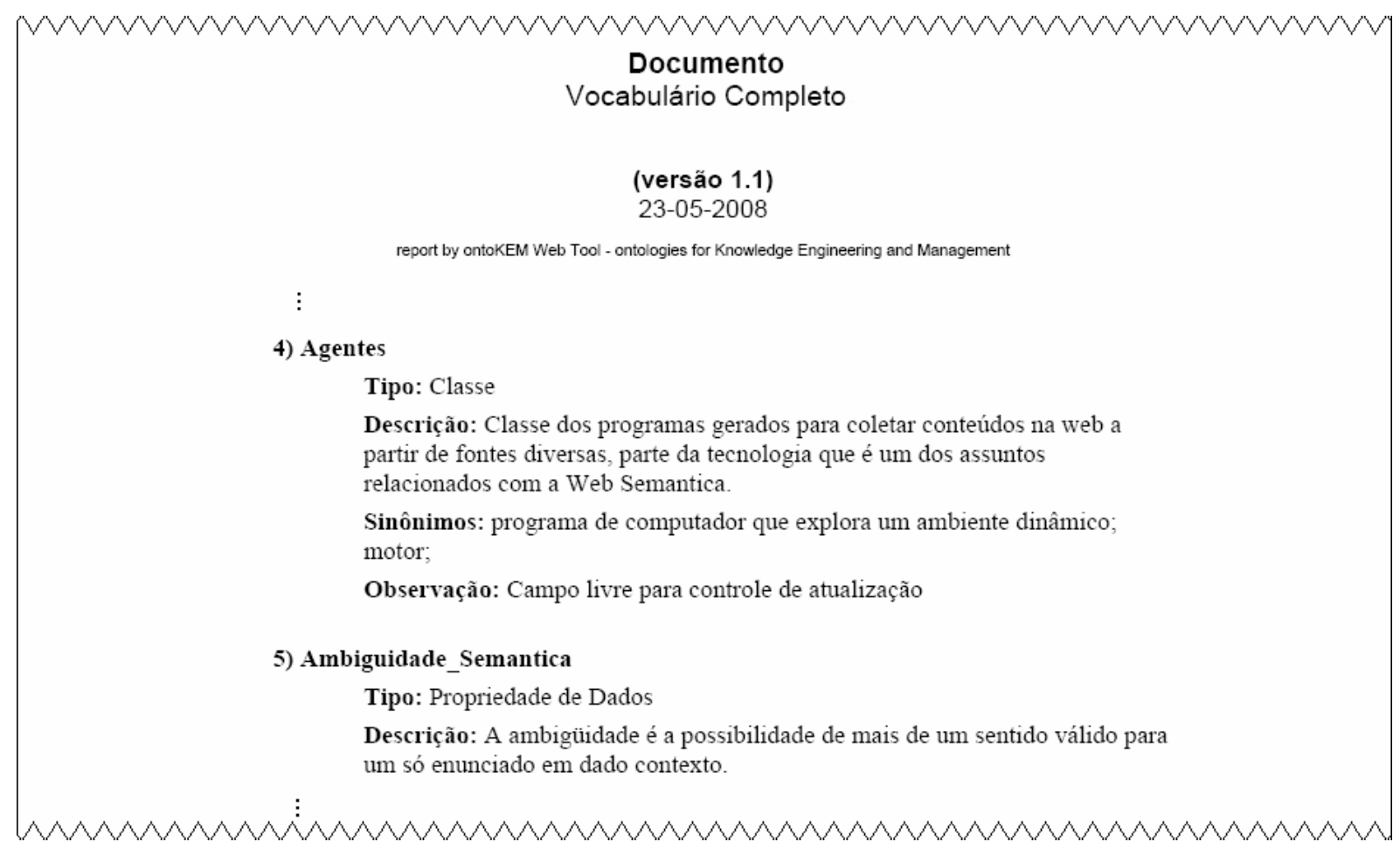

FIGURA 7 - Representação do documento "Vocabulário Completo"

Fonte: adaptado de MOLOSSI, 2008.

Uma vez definidas as classes, segundo as metodologias de desenvolvimento de ontologias, é possível definir a hierarquia de classes. Para essa atividade, a ontoKEM gera dois artefatos: o "Gráfico da Hierarquia de Classes" e, de forma descritiva, o documento "Hierarquia de Classes". Um gráfico de hierarquia de classes é exemplificado na FIG. 8.

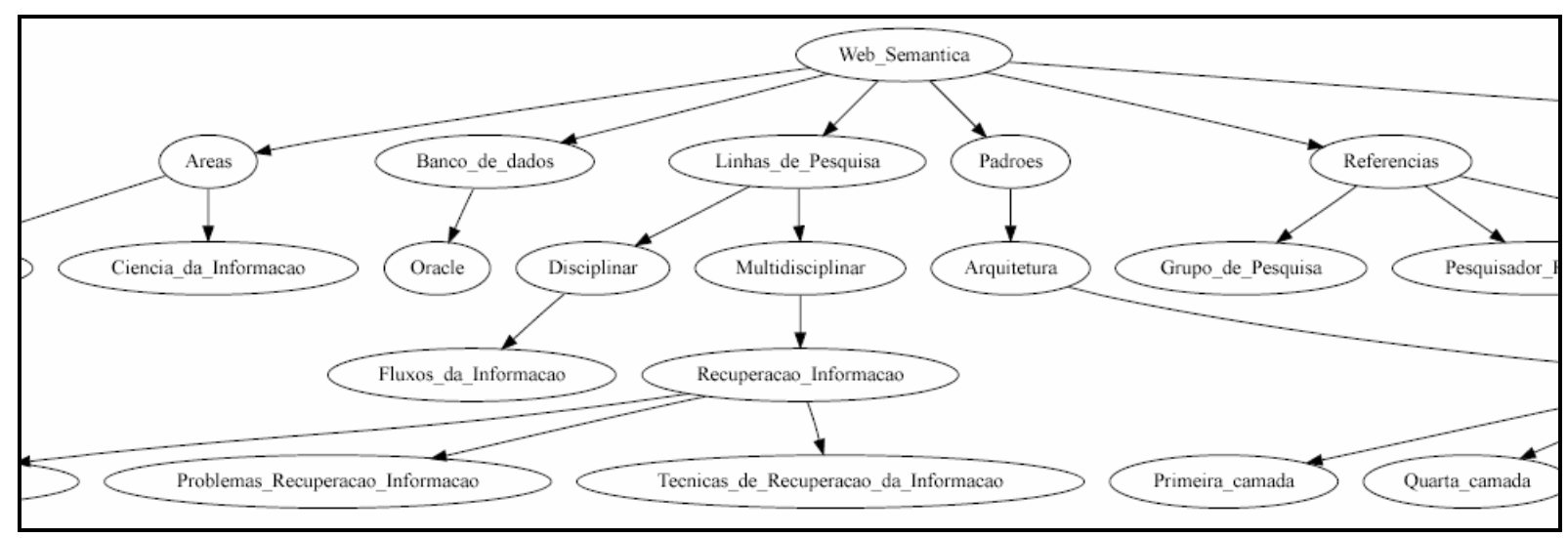

FIGURA 8: Representação gráfica da "Hierarquia de Classes"

Fonte: adaptado de MOLOSSI, 2008. 
Outro artefato de documentação importante gerado pela ontoKEM é o "Dicionário de Classes", sendo que um exemplo desse artefato é ilustrado na FIG. 9. No processo metodológico de desenvolvimento de ontologias, esse artefato é o resultado do mapeamento das relações, propriedades de classe, restrições e instâncias para cada classe da ontologia.

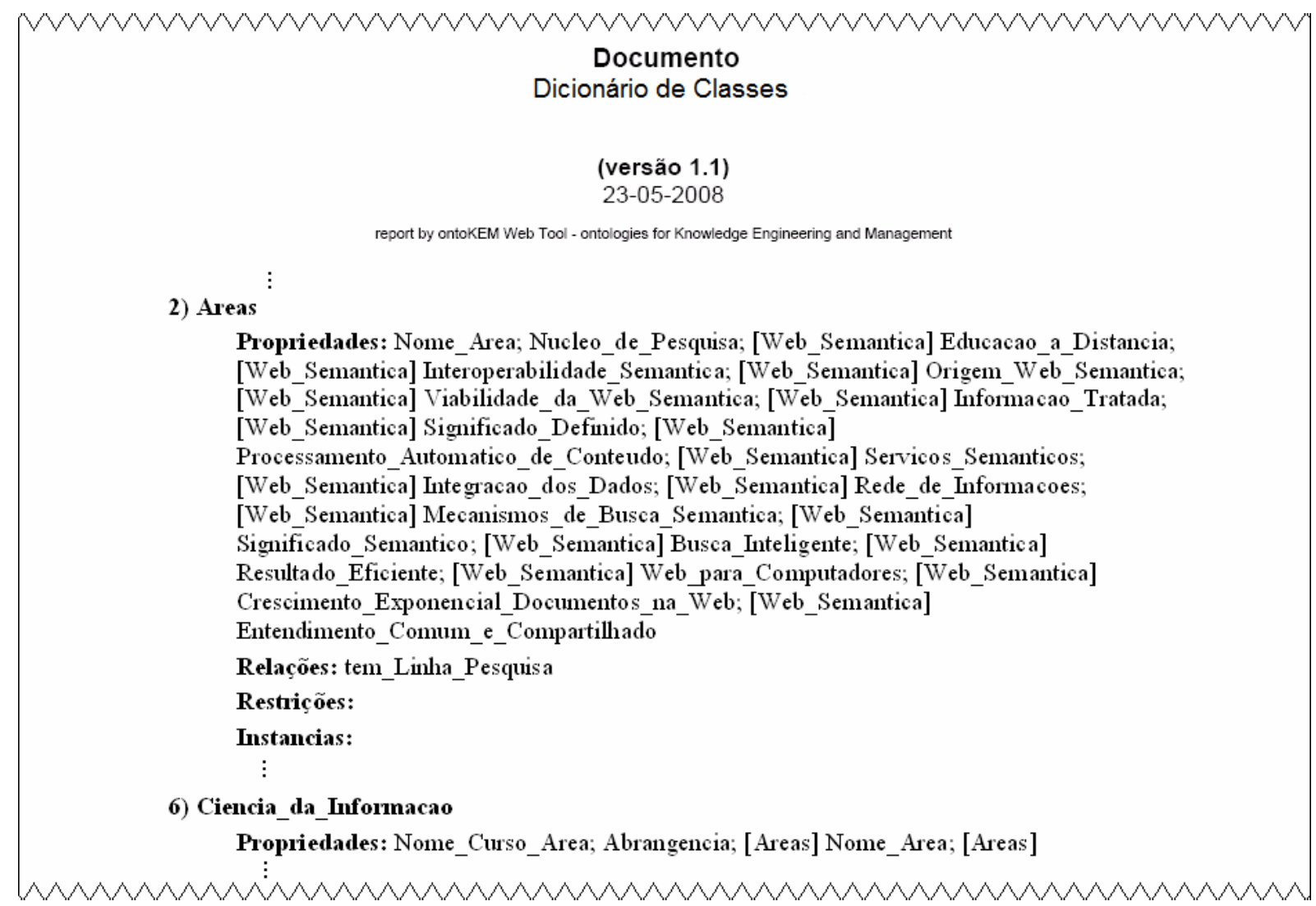

FIGURA 9 - Representação do documento "Dicionário de Classes"

Fonte: adaptado de MOLOSSI, 2008.

Um outro exemplo de prática, demonstrando os artefatos documentais gerados pela ontoKEM, pode ser acessado a partir do sítio "http://ontokem.egc.ufsc.br/ontologiaNavegadoresWeb/index.html". Nesse exemplo didático de uma ontologia de domínio, os autores relacionam os navegadores de Internet de código-aberto com as suas características funcionais (QUINAIA et al., 2008).

\subsection{Limitações da ontoKEM}

A ontoKEM foi desenvolvida para pesquisas experimentais em projetos de ontologias. Na versão atual da ferramenta, suas funcionalidades auxiliam somente as atividades de especificação da ontologia e aquisição do conhecimento, não compreendendo as atividades 
Ferramenta ontoKEM: uma contribuição à Ciência da Informação para o desenvolvimento de ontologia
Sandro Rautenberg; Antonio Costa Gomes Filho; José Leomar Todesco; Fernando Álvaro Ostuni-Gauthier

de implementação e de avaliação de ontologias, conforme pode ser percebido na FIG. 10.

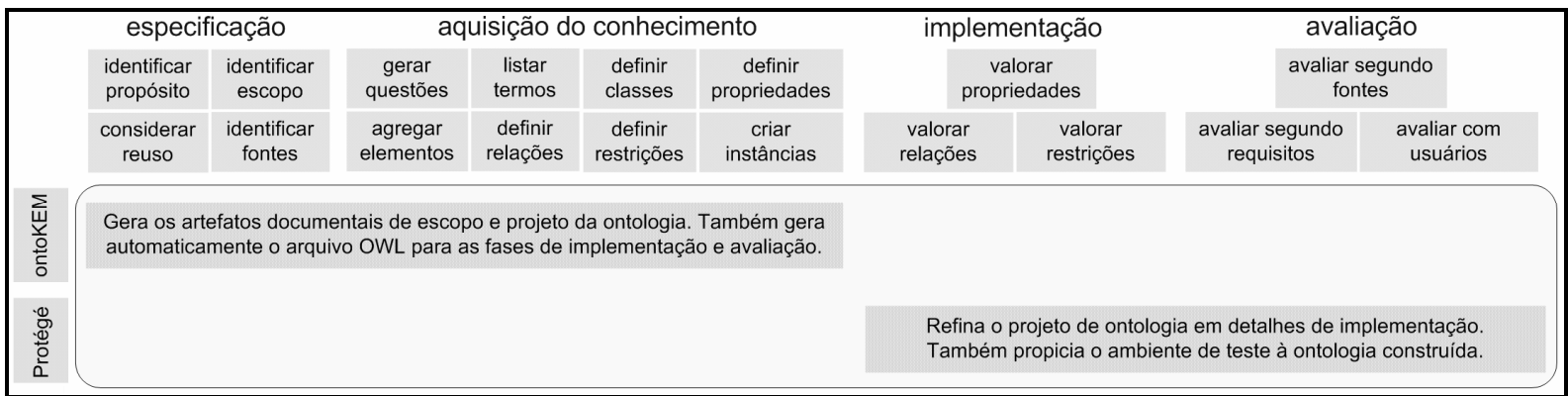

FIGURA 10 - OntoKEM e sua utilização nas atividades do ciclo de vida de ontologias

Fonte: Adaptado de RAUTENBERG; TODESCO; GAUTHIER (2009).

Contudo, para se contornar essa limitação, a ontoKEM oferece uma funcionalidade de exportação do projeto de ontologias através de arquivos no formato owl. Esses arquivos, por exemplo, podem ser importados em outros ambientes como o Protégé, permitindo o refinamento e avaliação dos projetos de ontologia. Parte de um arquivo .owl é demonstrada na FIG. 11.

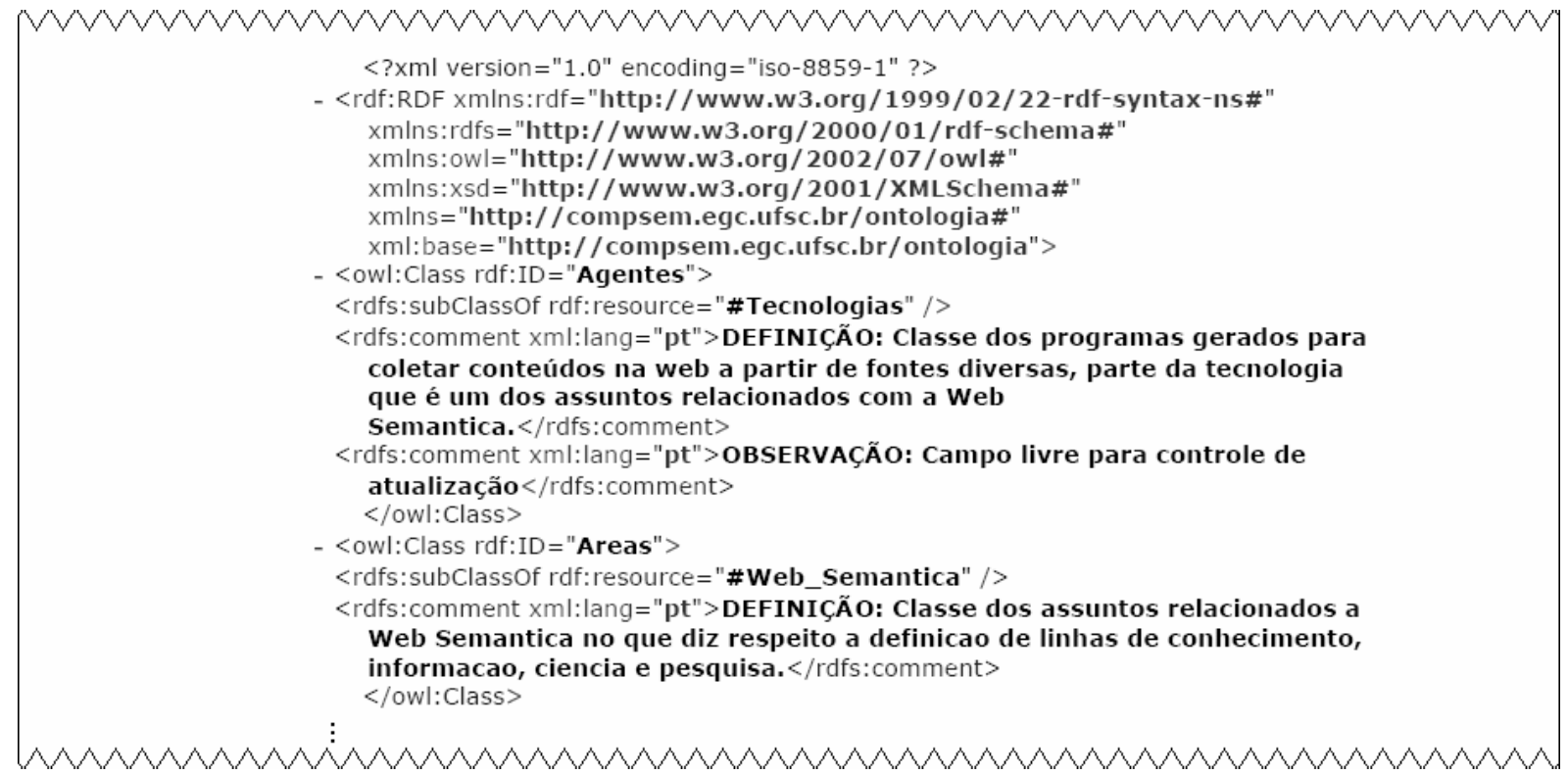

FIGURA 11 - Trecho de código-fonte de uma ontologia, transcrita na linguagem owl

Fonte: adaptado de MOLOSSI, 2008.

Outras limitações da ontoKEM podem ser atribuídas às funcionalidades de importação de projetos existentes (reutilização), de copiar e de restaurar projetos de ontologias. 
No entanto, mesmo considerando-se a gama de artefatos de documentação e a incorporação de um método de desenvolvimento de ontologias, como características principais da ferramenta, vê-se com "bons olhos" a utilização da ontoKEM em pesquisas aplicadas e/ou exploratórias.

A próxima seção apresenta as pesquisas experimentais em nível acadêmico já realizadas na área de $\mathrm{CI}$, com o emprego da ontoKEM.

\section{Casos de aplicação da ontoKEM na CI}

Acessando-se a ontoKEM, na condição de administrador do sistema, e tendo-se, assim, o acesso total à base de dados da ferramenta, verificou-se a existência de 83 usuários e 127 projetos de ontologias cadastrados. Dos projetos de ontologias cadastrados, observou-se que sete desses - aproximadamente $6 \%$ - contemplam ontologias utilizadas ou desenvolvidas no campo da CI. O Quadro 2 enumera o escopo dos referidos projetos.

QUADRO 2 Relação dos projetos de ontologia cadastrados na ontoKEM no domínio da CI.

\begin{tabular}{|c|c|}
\hline Projeto & Nome e Escopo \\
\hline 1 & $\begin{array}{l}\text { Nome: Preservação_de_documentos_digitais_dependência_de_HW_SW } \\
\text { Escopo: Ontologia para auxiliar o processo de preservação de documentos } \\
\text { digitais, mapeando quais os componentes de hardware e de software } \\
\text { necessitam ser mantidos na organização para possibilitar a visualização dos } \\
\text { mesmos. }\end{array}$ \\
\hline 2 & $\begin{array}{l}\text { Nome: Mapeamento_Tematico_de_Bases_de_Dados } \\
\text { Escopo: Mapear as áreas e sub-áreas de conhecimento de artigos científicos } \\
\text { publicados nos periódicos de uma Base de Dados. Esta ontologia será usada } \\
\text { para visualizar as áreas de conhecimento as quais pertencem os artigos } \\
\text { científicos, seus periódicos e permitindo o mapeamento temático das } \\
\text { publicações, relacionando também as pessoas (autorias) em seus espaços de } \\
\text { atuação. }\end{array}$ \\
\hline 3 & $\begin{array}{l}\text { Nome: COMUNICACAO_CIENTIFICA_referências } \\
\text { Escopo: Devido à importância da área da comunicação científica, em especial } \\
\text { os periódicos científicos on-line, sua padronização e sua recuperação de forma } \\
\text { rápida, com qualidade e relevante, optou-se por desenvolver uma ontologia } \\
\text { para a recuperação de informações científicas, publicados em periódicos } \\
\text { científicos, anais de eventos conceituados e livros técnico-científicos. A } \\
\text { informação científica é um dos insumos mais importante para o } \\
\text { desenvolvimento científico e tecnológico dos países, portanto veículo } \\
\text { disseminador e promovedor da evolução do conhecimento. }\end{array}$ \\
\hline 4 & $\begin{array}{l}\text { Nome: Biblioteconomia } \\
\text { Escopo: Aborda o domínio da Biblioteconomia }\end{array}$ \\
\hline
\end{tabular}




\begin{tabular}{|c|c|}
\hline eto & \\
\hline 5 & $\begin{array}{l}\text { Nome: web_semântica } \\
\text { Escopo: Essa ontologia classifica os principais componentes e elementos } \\
\text { relacionados ao domínio da web semântica. }\end{array}$ \\
\hline 6 & $\begin{array}{l}\text { Nome: WebSemântica } \\
\text { Escopo: A ontologia da Web Semântica pretende atender a demanda de } \\
\text { termos e conceitos compreendidos nos metadados Título, Assunto e Resumo } \\
\text { das teses e dissertações existentes na base de dados e aplicação da BDTD, do } \\
\text { IBICT. O domínio compreende os documentos existentes na interseção dos } \\
\text { assuntos "web semântica" e "recuperação da informação". Quanto à função e } \\
\text { ao tipo da ontologia, deverá ser considerada uma ontologia de domínio. O grau } \\
\text { de formalismo deverá discorrer sobre uma ontologia semi-informal. No que diz } \\
\text { respeito à profundidade ontológica a ontologia deverá compreender o nível de } \\
\text { taxonomia, onde vocabulário e significado são estabelecidos pela definição dos } \\
\text { relacionamentos hierárquicos. }\end{array}$ \\
\hline 7 & $\begin{array}{l}\text { Nome: Comunidades_informacionais } \\
\text { Escopo: Nesse projeto, aborda-se a formação de comunidades informacionais, } \\
\text { como opção de governo eletrônico e sua interação com os cidadãos dando } \\
\text { dinamicidade a estas relações e maior possibilidade de resolução de problemas } \\
\text { a partir da utilização de ciências cognitivas e inteligência artificial em prol da } \\
\text { democracia. O Governo Eletrônico conceitualmente baseia-se no uso da } \\
\text { tecnologia da informação para auxiliar as ações governamentais em suas } \\
\text { diferentes instâncias, G2G, G2C, G2B. Nesse sentido, as ações no âmbito do } \\
\text { governo para o cidadão encontram-se bastante limitadas a sites informacionais } \\
\text { e prestação de serviços on-line, considerando que as tecnologias emergentes e } \\
\text { disponíveis possibilitam um leque bastante amplo de aplicações indo ao } \\
\text { encontro dos princípios do e-Gov. As comunidades informacionais se } \\
\text { apresentam como uma das possíveis formas de captar a opinião do cidadão. } \\
\text { Esta interação vai construir informações que embasem políticas públicas de } \\
\text { acordo com os anseios e necessidades dos cidadãos, bem como transparência } \\
\text { das ações. Comunidades informacionais em e-Gov na web trata-se de um } \\
\text { ambiente web, de acesso gratuito onde serão estabelecidas comunidades para } \\
\text { discussão de assuntos de interesse comum através da criação de ontologias. } \\
\text { Serão criados fóruns sobre temáticas governamentais, enquetes e plebiscitos } \\
\text { informais, bem como permitirá o monitoramento das ações do governo, pois a } \\
\text { ferramenta tem como fontes de informação, órgãos governamentais de mídia } \\
\text { eletrônica e fontes digitais comunitárias e livres. }\end{array}$ \\
\hline
\end{tabular}

Fonte: OntoKEM (2009) - base de dados.

Ressalta-se que os projetos de ontologias anteriormente relacionados foram desenvolvidos no âmbito de working papers, como requisito de avaliação à disciplina de Tópicos Especiais em Engenharia do Conhecimento - Desenvolvimento de Ontologias, ministrada no Programa de Pós-Graduação em Engenharia e Gestão do Conhecimento da Universidade Federal de Santa Catarina. O projeto de ontologia 7 comunidades informacionais - descrito no quadro 2 é parte dos resultados da dissertação de Molossi (2008). 


\section{Conclusão}

Neste artigo apresentou-se a ferramenta ontoKEM como uma contribuição à CI para construção de projetos de ontologias. A ontoKEM é uma ferramenta case baseada na web concebida, em especial, para fins de documentação e construção de projetos de ontologias em pesquisas aplicadas. No artigo foram destacadas as características da ferramenta, quanto ao processo metodológico de desenvolvimento de ontologias e a riqueza de artefatos de documentação empregados pela ferramenta.

Perante as características da ontoKEM, e mediante a apresentação de exemplos de projetos de ontologias voltados ao domínio da $\mathrm{CI}$, concluise que a ontoKEM serve aos propósitos de organizar e representar a informação, os quais são atividades da prática da CI.

Contudo, a ontoKEM também apresenta algumas limitações quanto a todo o ciclo de vida de uma ontologia (especificação, aquisição do conhecimento, implementação e avaliação), não contemplando as atividades de implementação e avaliação de ontologias.

Não obstante às limitações, destaca-se que as vantagens de uso da ferramenta são que ela é de uso livre, portanto, sem custos para o usuário. Destaca-se também que para se contornar as limitações, a ontoKEM permite a exportação de projetos de ontologias via arquivos owl, os quais podem ser importados para o PROTEGE, por exemplo.

Por isso, é de interesse dos pesquisadores envolvidos com a ferramenta, a evolução da mesma, no sentido de abarcar as atividades ainda não contempladas, de forma que a massificação da utilização da ferramenta permitirá evoluir para questões relacionadas à sua comunicabilidade e usabilidade. Nesse sentido, a utilização da ontoKEM por pesquisadores e profissionais da CI é uma importante forma de colaboração, de forma que aqui fica o incentivo ao seu uso.

\section{Referências}

ALMEIDA, M. B. Roteiro para construção de uma ontologia bibliográfica através de ferramenta automatizada. Perspectivas em Ciência da Informação, v. 8, n. 2, p. 164-179, 2003.

ALMEIDA, M. B. Um modelo baseado em ontologias para representação da memória organizacional. Tese (Doutorado), Programa de Pós-Graduação em Ciência da Informação, Escola de Ciência da Informação, Universidade Federal de Minas Gerais - UFMG, 2006.

ALMEIDA, M. B.; BAX, M. P. Uma visão geral sobre ontologias: pesquisa sobre definições, tipos, aplicações, métodos de avaliação e de construção. Ciência da Informação, v. 32, n. 3, p. 7-20, 2003.

BRUSA, G. et al. Towards ontological engineering: a process for building a domain ontology from scratch in public administration. Expert Systems, v. 25, n., 5, p. 484-503, 2008. 
CAMPOS, M. L. de A. et al. Organização de unidades de conhecimento em hiperdocumentos: o modelo conceitual como espaço comunicacional para a realização da autoria. Ciência da Informação, v. 32, n. 2, p. 7-16, 2003.

$\mathrm{CORCHO}, \mathrm{O}$. et al. Methodologies, tools and languages for building ontologies. Where is their meeting point? Data \& Knowledge Engineering, v. 46, n. 1 , p. 41-64, 2003

DACONTA, M. C.; OBRST, L. J.; SMITH, K. T. The semantic web: a guide to the future of $\mathrm{xml}$, web services, and knowledge management. Indianapolis: Wiley Publishing, 2003.

FENSEL, D.; HERMELEN, F. V. On-to-knowledge: content-driven knowledge management tools through evolving ontologies. Disponível em: http://www.ontoknowledge.org/downl/del33.pdf. Acesso em: 02 abr. 2008.

FERNÁNDEZ-LÓPEZ, M.; GÓMEZ-PÉREZ, A. Overview and analysis of methodologies for building ontologies. The Knowledge Engineering Review, v. 17, n. 2, p. 129-156, 2002.

GASEVIC, D; DJURIC, D.; DEVED, V. Model driven architecture and ontology development. Heidelberg: Springer-Verlag, 2006.

GÓMEZ-PÉREZ, A. et al. Ontological Engineering: with examples from the areas of knowledge management, e-commerce and the semantic web. London: Springer-Verlag, 2004.

GOMES FILHO, A. C.; DAMASIO, E.; MIRANDA, P. C. O domínio da Ciência da Informação: problemas na conceitualização e na formação de consenso. In: XIX Congresso Brasileiro de Biblioteconomia e Documentação, 2000, Porto Alegre. Anais... Porto Alegre: PUC, 2000. v. 1 , p. $1-12$.

GOTTSCHALG-DUQUE, C. SiRILiCO: uma proposta para um sistema de recuperação de informação baseado em teorias da linguística computacional e ontologia. Tese (Doutorado), Programa de PósGraduação em Ciência da Informação, Escola de Ciência da Informação, Universidade Federal de Minas Gerais - UFMG. 2005.

IEEE. IEEE Guide for Software Quality Assurance Planning. [s.l. : s. n.], 1995.

ISKE, P.; BOERSMA, W. Connected brains: Question and answer systems for knowledge sharing: concepts, implementation and return on investment. Journal of Knowledge Management, v. 9, n. 1, p. 126-145, 2005.

KIRYAKOV, A. Ontologies for knowledge management. In: DAVIES, J. et al. (Eds). Semantic web technologies: trends and research in ontologybased systems. Chichester: John \& Wiley, 2006. p. 115-138. 
LIAO, S. Knowledge management technologies and applications: literature review from 1995 to 2002. Expert Systems with Applications, v. 25, n. 2, p. $155-164,2003$.

MARCONDES, C. H. et al. Ontologias como novas bases do conhecimento científico. Perspectivas em Ciência da Informação, v. 13, n. 3, p. 20-39, 2008.

MIKA, P.; AKKERMANS, $H$. Towards a new synthesis of ontology technology and knowledge management. The Knowledge Engineering Review, v. 19, n. 4, p. 317-345, 2005.

MOLOSSI, S. Inserção da biblioteca digital de teses e dissertações no contexto da web semântica: construção e uso de ontologias. Dissertação (Mestrado), Programa de Pós-Graduação em Ciência da Informação, Universidade Federal de Santa Catarina - UFSC. 2008.

MOREIRA, A. Uso de ontologia em sistemas de informação computacionais. Perspectivas em Ciência da Informação, v. 7, n. 1, p. 4960, 2002.

NOY, N. F.; MCGUINNESS, D. L. Ontology Development 101: a guide to creating your first ontology. Disponível em: <http://wwwksl.stanford.edu/people/dlm/papers/ontology-tutorial-noymcguinness.pdf>. Acesso em: 02 abr. 2008.

ONTOKEM.

Disponível

em: <http://ontokem.egc.ufsc.br/ProjetoOntologias/index.html>. Acesso em: 31/mar.2009.

PICKLER, M. E. V. Web Semântica: ontologias como ferramentas de representação do conhecimento. Perspectivas em Ciência da Informação, v. 12, n. 1, p. 65-83, 2007.

PINTO, H. S.; MARTINS, J. P. Ontologies: how can they be built? Knowledge and Information Systems, v. 6, n. 4, p. 441-464, 2004.

QUINÁIA, M. A. et al. Uma Ontologia de Domínio para Navegadores Web. In: CONGRESSO DE TECNOLOGIAS PARA GESTÃO DE DADOS $E$ METADADOS DO CONE SUL CONGED, 6., 2008, Curitiba. Anais... Curitiba: UFPR, 2008.

RAUTENBERG, $S$. et al. OntoKEM: uma ferramenta para construção e documentação de ontologias. In: SEMINÁRIO DE PESQUISA EM ONTOLOGIA NO BRASIL, 1., 2008, Niterói. Anais... Niterói: UFF, 2008.

RAUTENBERG, S.; TODESCO, J. L.; GAUTHIER, F. A. O. Processo de desenvolvimento de ontologias: uma proposta e uma ferramenta. Revista Tecnologia, v. 30, n. 1, p. 133-144, jun. 2009.

RELATÓRIO FINAL - ANO I. Gestão da rede de distribuição de média tensão empregando ontologias de domínio e sistemas de conhecimento LEC-EGC-UFSC. Florianópolis, 2008. Relatório. Mimeografado 
SURE, Y.; STUDER, R. A Methodology for ontology-based Knowledge Management. In: DAVIES, J. et al. (Eds). Towards the semantic web: ontology-driven knowledge management. Chichester: John Wiley \& Sons, 2003. p. 33-46.

USCHOLD, M. Knowledge level modelling: concepts and terminology. The Knowledge Engineering Review, v. 13, n. 1, p. 5-29, 1998. 